\title{
Twenty Years of Radiation Therapy of Unresectable Intrahepatic Cholangiocarinoma: Internal or External? A Systematic Review and Meta-Analysis
}

\author{
Qian $\mathrm{Yu}^{\mathrm{a}}$ Chenyu Liu $^{\mathrm{b}}$ Anjana Pillai ${ }^{\mathrm{c}}$ Osman Ahmed ${ }^{\mathrm{d}}$ \\ aDepartment of Surgery, Cleveland Clinic Florida, Weston, FL, USA; ${ }^{b}$ Ben May Department of Cancer Research, \\ University of Chicago, Chicago, IL, USA; 'Division of Gastroenterology, Hepatology, and Nutrition, University of \\ Chicago, Chicago, IL, USA; 'Division of Interventional Radiology, Department of Radiology, University of Chicago, \\ Chicago, IL, USA
}

\section{Keywords \\ Cholangiocarcinoma $\cdot$ Radioembolization - Radiation therapy · Interventional radiology · Radiation oncology}

\begin{abstract}
Purpose: Both external beam radiation therapy (EBRT) and selective-internal radiation therapy (SIRT) are implemented to treat unresectable intrahepatic cholangiocarcinoma (iCCA). The present study aimed to evaluate the efficacy of EBRT and SIRT in managing iCCA through a systematic review and meta-analysis. Methods: PubMed and Cochrane database were queried to search for studies published from January 2000 toJune 2020 without language restrictions. Median survival time, overall survival, and radiological response were extracted. Secondary outcomes such as complication rates, predictors of survival, and downstage to surgery were pooled. Patient-level survival data were obtained to generate Kaplan-Meier survival graph. Pooled outcomes were analyzed with a random-effect model. Results: Twenty-nine and 20 studies including 732 and 443 patients from the SIRT and EBRT groups were included in the present study. From initial radiation treatment, the median survival time for patients who underwent SIRT and EBRT were 12.0 (95\% confidence interval [CI]: 10.8-14.6) and 13.6 (95\% Cl: 11.1-16.0)
\end{abstract}

months, respectively. As first-line therapy, the median survival time was 36.1 (95\% Cl: 20.6-39.5) months for SIRT and 11.0 (95\% Cl: 9.3-13.6) months for EBRT. Both radiation modalities were effective in downstaging initially unresectable iCCA to surgery (SIRT: $30.5 \%$; EBRT: 18.3\%). Patients in the SIRT group encountered more post-embolization abdominal pain (6.9 vs. $2.2 \%)$, ulcer (1.0 vs. $0.5 \%)$, nausea (1.6 vs. $0.7 \%$ ), anorexia ( 5.9 vs. $0 \%$ ), thrombocytopenia (7.3 vs. $6.0 \%$ ), hyperbilirubinemia (5.2 vs. $2.1 \%$ ), and hypoalbuminemia (13.2 vs. $3.3 \%)$, whereas EBRT was associated with higher rates of anemia ( $0.6 \mathrm{vs.} 7.5 \%$ ) and neutropenia ( $6.5 \mathrm{vs.} 11.0 \%$ ). Conclusions: Both EBRT and SIRT were safe and effective in treating unresectable iCCA. However, available evidence was highly heterogeneous regarding patient population, limiting fair comparison between 2 radiation modalities. Future high-quality comparative studies are warranted.

(c) 2021 The Author(s).

Published by S. Karger AG, Basel

\section{Introduction}

Intrahepatic cholangiocarinoma (iCCA) is a primary hepatic malignancy arising from peripheral intrahepatic bile ducts [1]. While rare, a notable increasing trend has been observed worldwide in terms of incidence and mor-
(C) 2021 The Author(s)

Published by S. Karger AG, Basel

This is an Open Access article licensed under the Creative Commons Attribution-NonCommercial-4.0 International License (CC BY-NC) (http://www.karger.com/Services/OpenAccessLicense), applicable to the online version of the article only. Usage and distribution for commercial purposes requires written permission.
Correspondence to:

Qian Yu, yuqian1006@gmail.com 
tality [2-4]. Clinically, iCCA typically follows an indolent progression with nonspecific symptoms, presenting as advanced disease upon initial diagnosis [5]. While surgical resection offers potentially curative treatment to iCCA, $63-85 \%$ is considered unresectable at the time of diagnosis $[1,6]$. In addition, postoperative recurrence is frequent during follow-up, with a dismal 5-year survival of $20-35 \%$ [7-10]. For patients with locally advanced and unresectable iCCA, in addition to traditional systemic chemotherapy, liver directed therapy options including embolotherapy, radiotherapy, and direct hepatic arterial infusion all demonstrate promising data [11]. Efficacy and superiority of these treatments are however challenged by a lack of randomized-controlled trials.

Within the last decade, a growing amount of evidence has been published favoring the use of radiotherapeutic modalities for iCCA [12-15]. According to the International Liver Cancer Association and National Comprehensive Cancer Network guidelines, both selective-internal radiation therapy (SIRT) and external beam radiation therapy (EBRT) are acceptable approaches for locally advanced and unresectable iCCA $[11,16]$. A recent study utilizing the National Cancer Database, suggested an increased overall survival (OS) benefit with stereotacticbody radiation therapy (SBRT) over conventional chemoradiation (CRT) and transarterial radioembolization [17]. However, this study had several methodological limitations including significantly different baseline characteristics, small sample size (87 patients in the propensity-weighed analysis), and lack of data on treatment-related toxicities. Current recommendations for EBRT versus SIRT are not bolstered by high-quality evidence. Therefore, the purpose of the present study was to characterize the safety and efficacy of EBRT and SIRT for the treatment of iCCA through a systematic review and metaanalysis.

\section{Methods}

\section{Literature Screening}

The study was performed in accordance with the Preferred Reporting Items for Systematic Review and Meta-Analysis guideline. PubMed and Cochrane Library were queried from establishment to June 2020 without language restriction. The following terms were used:

1. "cholangiocarcinoma" AND ("radioembolization" OR" yttrium") for radioembolization;

2. "cholangiocarcinoma" AND ("radiation" OR "radiotherapy") for external radiation therapy.

The following inclusion criteria were adopted: (a) patient was diagnosed with iCCA and received EBRT or SIRT; (b) clinical fol- low-up data including radiological response and/or survival rates were reported. A study was excluded if the following criteria were met: (a) nonhuman studies; (b) case report and study with sample size $\leq 5$ patients; (c) absence of original data (letter, editorial, commentary, and review); (d) population-level study; (e) clinical outcomes were not selectively reported for patients with iCCA and received SIRT or EBRT; (f) studies published prior to the year of 2000; (g) studies solely focusing on adjuvant RT for post-resection iCCA; and (h) studies focusing on mixed-type HCC-CCA.

Endnote X8 (Clarivate Analytics, Philadelphia, PA) was used to identify and remove duplicates. Articles were initially screened based on titles, abstracts, and keywords, followed by a comprehensive review of full text of the remaining studies. Literature pertaining to RE and RT was queried and screened separately. A detailed screening process is depicted in online suppl. Figure 1 (for all online suppl.material, see www.karger.com/doi/10.1159/000516880).

\section{Data Acquisition}

The primary end points of interest were median survival time (MST), OS, and degree of radiographic response. Secondary end points were treatment-related adverse events, predictor analysis of OS (hazard ratio [HR]), and ability to downstage to surgery. In addition, the following baseline characteristics were retrieved: author, year, country, study design, treatment modality (EBRT vs. SIRT), sample size, age, sex, tumor number, tumor size, Eastern Cooperative Oncology Group Status (ECOG) performance, metastasis status, unilobar versus bilobar disease, tumor burden, infiltrative versus peripheral type, presence of cirrhosis, previous treatment history, radiation dosage, pulmonary lung fraction, the presence of portal venous invasion (PVI), and activity prescription method. Information regarding regions and institutions where studies were conducted was collected the last to minimize bias.

Measures of radiographic response, classified as complete response (CR), partial response (PR), stable disease (SD), and progressive disease $(\mathrm{PD})$, were extracted along the imaging evaluation methods. Response rate (RR) was defined as the combined rates of $\mathrm{CR}$ and PR; disease control rate (DCR) was defined as the combined rates of $\mathrm{CR}, \mathrm{PR}$, and $\mathrm{SD}$.

The MST and OS at 6 months, 1 year, 2 years, and 3 years were extracted. Individual patient survival outcomes were retrieved from survival curves of the original studies using GetGraph Digitizer v 2.26 (http://getdata-graph-digitizer.com/). Studies that did not label censored data (tick) were excluded from this analysis, as the survival outcomes of these patients could not be derived. Reasons of exclusion were tabulated (online suppl. Tables 1.1, 1.2). OS rate was pooled on Kaplan-Meier survival curve. MST and cumulative survival rates at 6 months, 1 year, 2 years, and 3 years were calculated.

The quality of each study was assessed with the methodological index for nonrandomized studies (online suppl. Table 2). Two authors retrieved the data independently. Any disagreement was resolved upon discussion.

\section{Statistical Analysis}

All quantitative analyses were performed with Stata 15.1 (STATA Corp., College Station, TX, USA). Meta-analysis was conducted with -metan and -metaprop one functions $[18,19]$. Radiological response, HR of predictive analysis, and adverse events were pooled and reported in weighted means with $95 \%$ Wald confidence intervals (CIs). A random-effect model was adopted due to 
the cross-study heterogeneity. Publication bias was analyzed with Egger's test and by assessing asymmetries on the funnel plot. For studies sharing overlapping patient sample, only the study with the largest sample size was included in the quantitative analysis for the variable of interest. Log-rank test was implemented to compare survival outcomes between groups. $p$ value $<0.05$ was considered significant.

\section{Results}

\section{Baseline Characteristics}

Among the initial 150 unique search results, 29 original studies from 17 groups of investigators were screened and considered in the meta-analysis of the SIRT [14, 2047] (online suppl. Fig. 1a). A total of 732 patients received SIRT (Table 1); the median/mean age ranged from 66.4 to 69.3 years old. The female versus male ratio, tumor number, distribution, size, and ECOG performance score of each study are specified in Table 1 . The median/mean percentage of extrahepatic metastasis at diagnosis, presence of cirrhosis, presence of PVI, and lung shunt fraction were $12.3-57.9,0-35,0-81.1$, and $4.4-7 \%$, respectively. The mean/median delivered radiation activity and dosage ranged from 1.20 to $1.76 \mathrm{GBq}$ and $92.7-322 \mathrm{~Gy}$, respectively. Among 9 studies that reported tumor burden relative to liver parenchyma, the total percentage of patients with $<25 \%$ tumor burden was 111/272 (40.8\%). Most patients received another form of anticancer treatment prior to the SIRT (Table 1). Only 2 studies $(2 / 17)$ focused solely on treatment-naive patients $(11.8 \%)$. Concomitant chemotherapy was administered in all enrolled patients in $5 / 12$ studies $(41.7 \%)$.

Regarding EBRT, 20 unique studies from 15 institutions were included in the present meta-analysis [13, 4866] (online suppl. Fig. 1b). The following radiation modalities were used: 5 photon-based conventional dose radiotherapy (CRT), 5 photon-based SBRT, 2 proton therapy (PT), 1 carbon-ion radiotherapy, and 3 studies utilized both photon/proton EBRT (1 conventional dosed, 1 ablative, and 1 hypofractionated). A total of 443 patients received RT (Table 2); the mean/median age ranged from 57 to 76 years old. The female/male ratio, tumor number, size, ECOG performance, distribution, peripheral versus infiltrative type was listed in Table 2. Tumor burden was not reported. Only Tse et al. [63] reported the percentage of PVI at diagnosis (30\%). The median/mean BED ranged from 50.1 to $109.3 \mathrm{~Gy}$. Excluding studies that did not specify previous treatment history, only $7 / 16(43.8 \%)$ studies involved patients who were treatment-naive prior to RT (Table 2). Concomitant che-

iCCA: Radioembolization versus External Radiation Therapy motherapy was administered in all enrolled patients in $3 / 10$ studies $(70.0 \%)$.

\section{Radiological Response}

For SIRT, the pooled RR according to response evaluation criteria in solid tumors (RECIST), modified RECIST (mRECIST), European Association for the Study of the Liver (EASL), World Health Organization (WHO), and Positron Emission Tomography Response Criteria in Solid Tumors (PERCIST) were 22\% (95\% CI: $10-37 \%$ ), 35\% (95\% CI: 4-75\%), 82\% (95\% CI: 48-100\%), 24\% (95\% CI: 14-39\%), and 81\% (95\%: 63-95\%), respectively (Fig. 1a). The pooled DCR were 75\% (95\% CI: 65-84\%), 78\% (95\% CI: $64-89 \%$ ), 98\% (95\% CI: $88-100 \%$ ), $98 \%$ (95\% CI: 88-100\%), and 100\% (95\% CI: 93-100\%) based on RECIST, mRECIST, EASL, WHO, and PERCIST, respectively (Fig. 1b). The funnel plot did not suggest significant difference among RR (Egger's test: $p=0.276$ ) and DCR (Egger's test: $p=0.559$ ) based on RECIST criteria (online suppl. Fig. 2a, b). Among the studies reporting RECIST criteria, $I^{2}=89.9 \%(p<0.01)$ and $76.7 \%(p<0.01)$ for RR and DCR, respectively.

For EBRT, the pooled RR based on RECIST, mRECIST, and EASL were $31 \%$ (95\% CI: 12-54\%), 46\% (95\% CI: 3064), and 36\% (95\% CI: 22-51), respectively (Fig. 1c). The DCR was 83\% (95\% CI: 61-98\%), 89\% (95\% CI: 73-96\%), 84\% (95\% CI: 71-94\%) according to RECIST, mRECIST, and EASL, respectively (Fig. 1d). Based on RECIST criteria (online suppl. Fig. 2c, d), funnel plot did not suggest significant difference among RR (Egger's test: $p=0.407$ ) and DCR (Egger's test: $p=0.252)$. The RR of SBRT (48\%, 95\% CI: 26-70\%) was higher than CRT (31\%, 95\% CI: 19$43 \%$ ); the DCR of PT (99\%, 95\% CI: 95-100\%) was higher than SBRT (73\%, 95\% CI: 52-91\%) and CRT (71\%, 95\% CI: 36-92\%; online suppl. Fig. 3). Among the studies reporting RECIST criteria, $I^{2}=77.5 \%(p<0.01)$ and $83.9 \%$ $(p<0.01)$ for RR and DCR, respectively.

\section{Survival}

For patients who received SIRT, the MST of included studies ranged from 6.3 to 33.6 months (Table 3 ). OS at 6 months, 1 year, 2 years, and 3 years were $56-100$, 32.6$97.3,5-68.6$, and $0-46.2 \%$, respectively. In terms of perpatient analysis, survival data of 450 individual patients were successfully obtained from 12 studies (Fig. 2a). The MST was 12.0 (95\% CI: 10.8-14.6) months from the time of first SIRT treatment. The cumulative OS rates at 6 months, 1 year, 2 years, and 3 years were $74.7 \%$ (70.378.5\%), 52.3\% (47.4-57.0\%), 33.3\% (28.7-37.9\%), and $25.3 \%(21.1-29.6 \%)$, respectively.

Liver Cancer 2021;10:433-450 


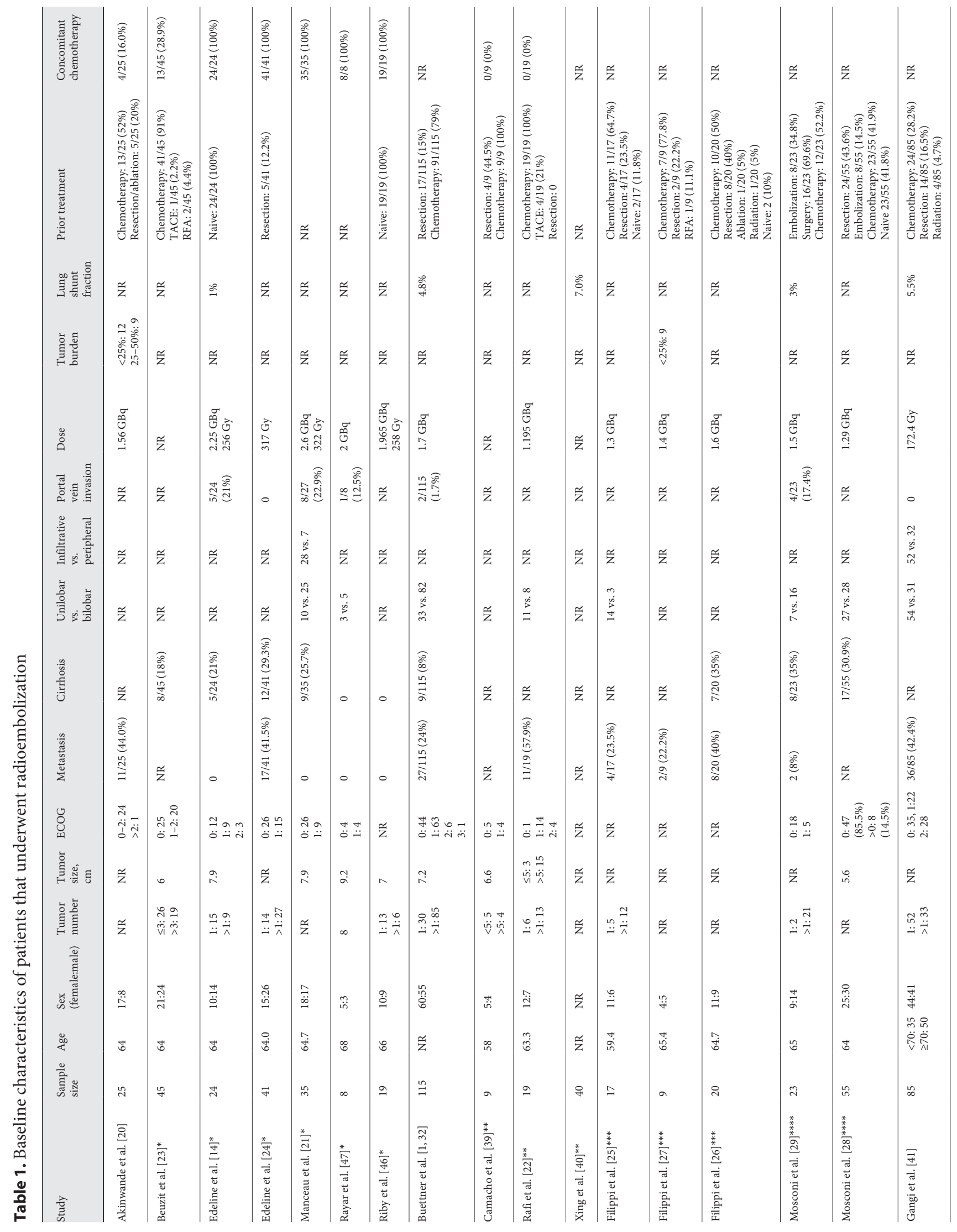




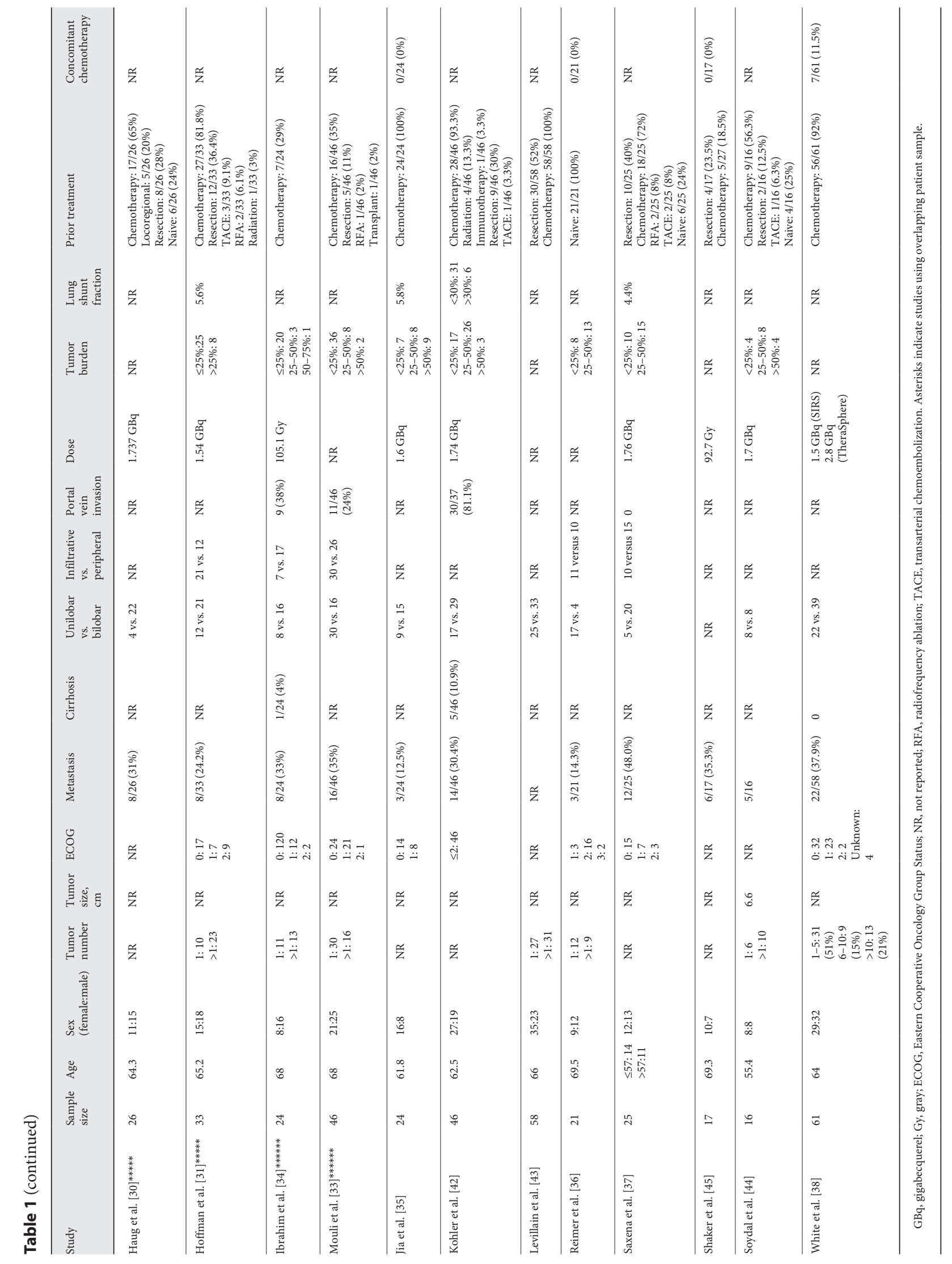




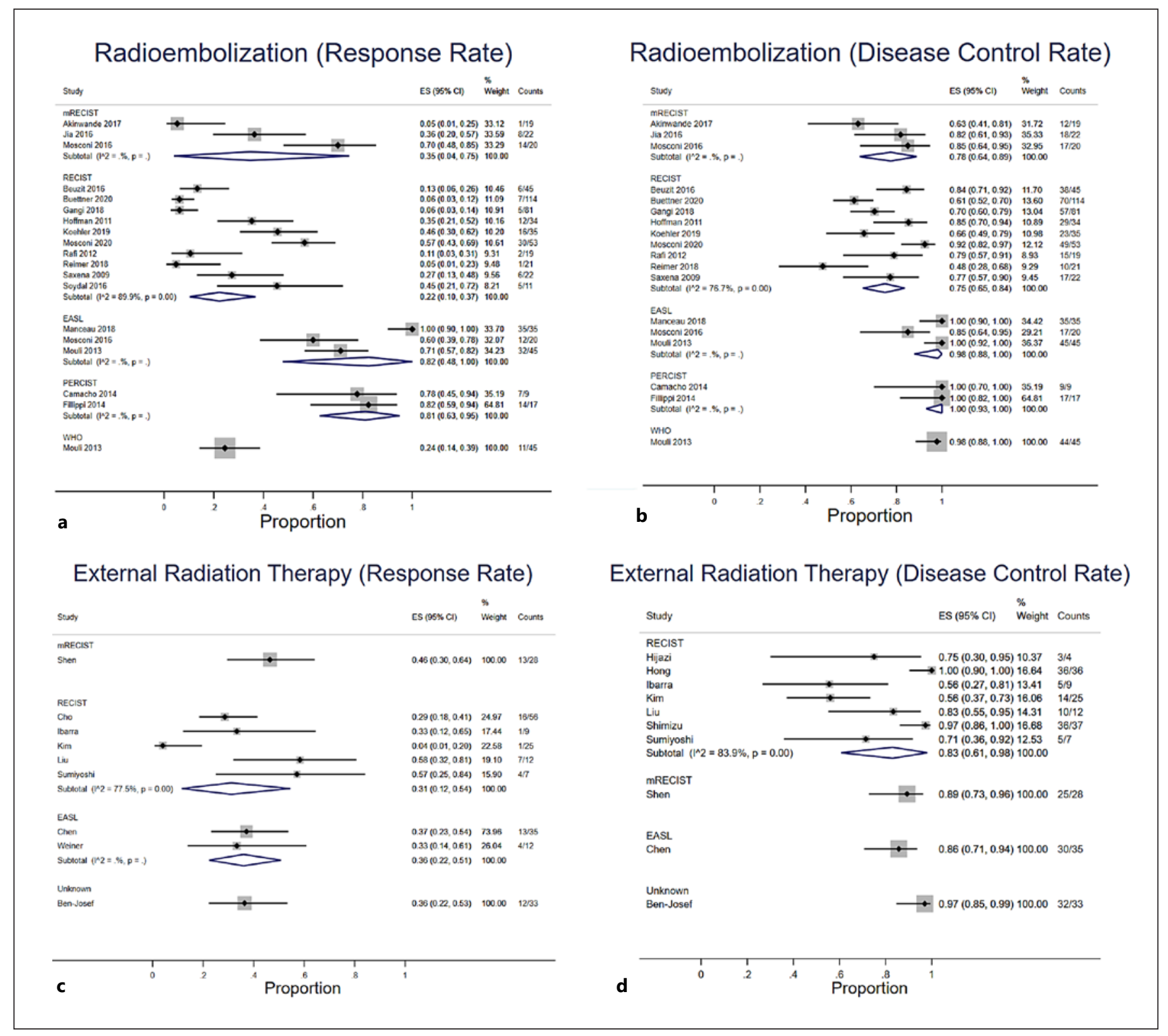

Fig. 1. Pooled RR and DCR. RR (a) and DCR (b) of SIRT. RR (c) and DCR (d) of EBRT. RR, response rate; DCR, disease control rate; SIRT, selective-internal radiation therapy; EBRT, external beam radiation therapy.

For patients who received EBRT, the MST ranged from 5.4 to 30 months. OS at 6 months, 1 year, 2 years, and 3 years were $67.2-98.7 \%, 30.4-87 \%, 0-61 \%$, and $0-44 \%$, respectively (Table 4). From the time of treatment, survival data of 272 patients were available from 10 studies (Fig. 2a). The MST was 13.6 (95\% CI: 11.1-16.0) months from the time of first EBRT treatment. The cumulative OS rates at 6 months, 1 year, 2 years, and 3 years were $76.2 \%$ (70.7-80.8\%), 49.8\% (43.8-55.6\%), $23.4 \%$
(18.6-28.6\%), and 9.5\% (6.4-13.4\%), respectively. Further differentiating by EBRT type (Fig. 2a), the MST of PT $(n=98)$, SBRT $(n=43)$, and CRT $(n=106)$ were 20.9 (95\% CI: 5.2-13.6), 13.1 (95\% CI: $10.0-21.1$ ), and 10.0 (95\% CI: 8.5-14.2) months, respectively. The 6-month, 1-year, 2-year, and 3-year OS of patients who underwent PT were 85.6 (95\% CI: 77.0-91.2), 66.3\% (95\% CI: 55.974.8), $44.9 \%$ (95\% CI: 34.4-54.9), and 31.4\% (95\% CI: 21.9-41.3), respectively, with an MST of 20.9 (95\% CI: 


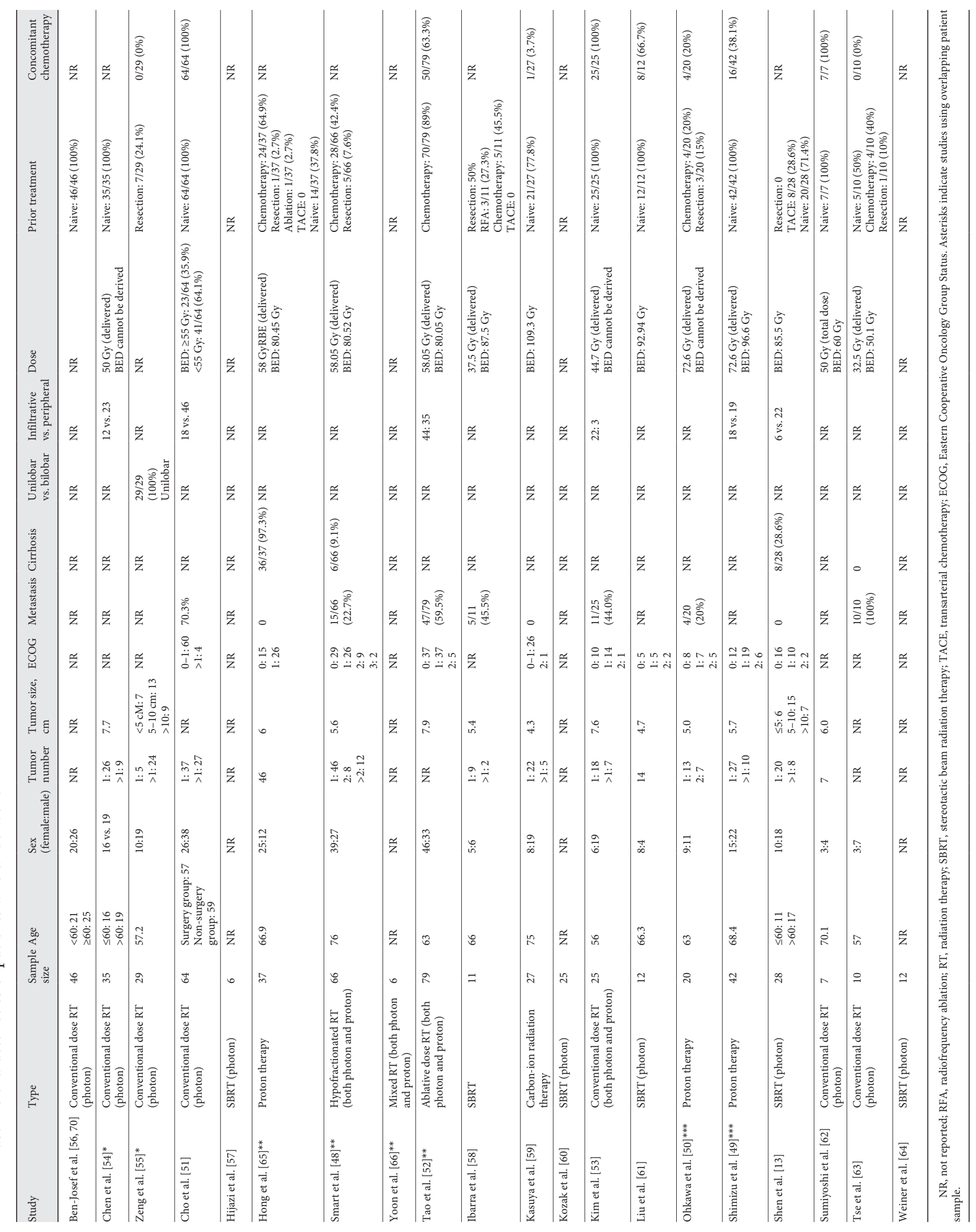


Table 3. Radiographical and survival outcomes of included studies on radioembolization

\begin{tabular}{|c|c|c|c|c|}
\hline Study & Criteria & $\begin{array}{l}\text { Radiological } \\
\text { Outcome }\end{array}$ & MST, months & OS \\
\hline Akinwande et al. [20] & mRECIST & $\begin{array}{l}\text { CR: } 1 / 19 \\
\text { PR: } 0 \\
\text { SD: } 11 / 19 \\
\text { PD: } 7 / 19\end{array}$ & NA & NA \\
\hline Edeline et al. [14]* & RECIST & Not shown & Not shown & Not shown \\
\hline Edeline et al. [24]* & RECIST & Not shown & Not shown & Not shown \\
\hline Riby et al. [46]* & RECIST & Not shown & Not shown & Not shown \\
\hline Camacho et al. [39]** & RECIST (also reported PERCIST) & Not shown & Not shown & Not shown \\
\hline Rafi et al. $[22]^{* *}$ & RECIST & $\begin{array}{l}\text { CR: } 0 \\
\text { PR: } 2 / 19 \\
\text { SD: } 13 / 19 \\
\text { PD: } 4 / 19\end{array}$ & Not shown & $\begin{array}{l}6 \text { mo: } 68.0 \% \\
1 \text { yr: } 48.5 \% \\
2 \text { yr: } 9.5 \% \\
3 \text { yr: NA }\end{array}$ \\
\hline Xing et al. $[40]^{* *}$ & NA & NA & $\begin{array}{l}\text { LSF }<10 \%: 26.1 \\
\text { LSF } \geq 10 \%: 16.1\end{array}$ & NA \\
\hline Buettner et al. [1, 32] & RECIST & $\begin{array}{l}\text { PR: } 7 / 96 \\
\text { SD: } 63 / 96 \\
\text { PD: } 26 / 96\end{array}$ & 11 & $\begin{array}{l}6 \text { mo: } 68.9 \% \\
1 \text { yr: } 44 \% \\
2 \text { yr: } 19.8 \% \\
3 \text { yr: } 4 \%\end{array}$ \\
\hline Filippi et al. [27]*** & PERCIST & Not shown & Not shown & Not shown \\
\hline Filippi et al. [26] $]^{* * *}$ & NA & NA & 12.5 & $\begin{array}{l}6 \text { mo: } 75 \% \\
1 \text { yr: } 40 \% \\
2 \text { yr: } 5 \% \\
3 \text { yr: NA }\end{array}$ \\
\hline Mosconi et al. [29] $]^{* * * *}$ & RECIST (also reported mRECIST and EASL) & Not shown & Not shown & Not shown \\
\hline Mosconi et al. [28]**** & RECIST & $\begin{array}{l}\text { CR: } 4 / 53 \\
\text { PR: } 26 / 53 \\
\text { SD: } 19 / 53 \\
\text { PD: } 4 / 53\end{array}$ & 16.7 & $\begin{array}{l}6 \text { mo: } 90.8 \% \\
1 \text { yr: } 59.4 \% \\
2 \text { yr: } 31.8 \% \\
3 \text { yr: NA }\end{array}$ \\
\hline Gangi et al. [41] & RECIST & $\begin{array}{l}\text { CR: } 0 \\
\text { PR: } 5 / 81 \\
\text { SD: } 52 / 81 \\
\text { PD:24/81 }\end{array}$ & 12 & $\begin{array}{l}6 \text { mo: } 70 \% \\
1 \text { yr: } 49 \% \\
2 \text { yr: } 38 \% \\
3 \text { yr: NA }\end{array}$ \\
\hline Haug et al. [30]***** & RECIST & Not shown & Not shown & Not shown \\
\hline
\end{tabular}


Table 3 (continued)

\begin{tabular}{|c|c|c|c|c|}
\hline Study & Criteria & $\begin{array}{l}\text { Radiological } \\
\text { Outcome }\end{array}$ & MST, months & OS \\
\hline Hoffman et al. $[31]^{* * * * *}$ & RECIST & $\begin{array}{l}\text { CR: } 0 \\
\text { PR: } 12 / 34 \\
\text { SD: } 17 / 34 \\
\text { PD: } 5 / 34\end{array}$ & 22 & $\begin{array}{l}6 \text { mo: } 84.0 \% \\
1 \text { yr: } 57.3 \% \\
2 \text { yr: } 42.0 \% \\
3 \text { yr: } 12.8 \%\end{array}$ \\
\hline Ibrahim et al. [34]****** & WHO & Not shown & Not shown & Not shown \\
\hline Jia et al. [35] & mRECIST & $\begin{array}{l}\text { CR: } 0 \\
\text { PR: } 8 / 22 \\
\text { SD: } 10 / 22 \\
\text { PD: } 4 / 22\end{array}$ & 9.0 & $\begin{array}{l}6 \text { mo: } 69.9 \% \\
1 \text { yr: } 32.6 \% \\
2 \text { yr: } 20.4 \% \\
3 \text { yr: NA }\end{array}$ \\
\hline Levillain et al. [43] & NA & NA & 6.3 & $\begin{array}{l}6 \text { mo: } 66.9 \% \\
1 \text { yr: } 40.0 \% \\
2 \text { yr: } 22.0 \% \\
3 \text { yr: } 11.2 \%\end{array}$ \\
\hline Reimer et al. [36] & RECIST & $\begin{array}{l}\text { CR: } 0 \\
\text { PR: } 1 / 21 \\
\text { SD: } 9 / 21 \\
\text { PD: } 11 / 21\end{array}$ & 15 & $\begin{array}{l}6 \text { mo: } 100 \% \\
1 \text { yr: } 93.7 \% \\
2 \text { yr: } 68.6 \% \\
3 \text { yr: } 46.2 \%\end{array}$ \\
\hline Soydal et al. [44] & NA & NA & 9.8 & $\begin{array}{l}6 \text { mo: } 56.3 \% \\
1 \text { yr: } 32.8 \% \\
2 \text { yr: } 10.9 \% \\
3 \text { yr: NA }\end{array}$ \\
\hline White et al. [38] & NA & NA & 13.9 & $\begin{array}{l}6 \text { mo: } 68.7 \% \\
1 \text { yr: } 37.4 \% \\
2 \text { yr: } 7.5 \% \\
3 \text { yr: NA }\end{array}$ \\
\hline
\end{tabular}

CR, complete response; EASL, European Association for the Study of the Liver; MST, median survival time; NA, not reported by original study; Not shown, data were not displayed in the table because the outcome was reported by another larger study with overlapping patient sample; OS, overall survival; PD, progressive disease; PERCIST, positron emission tomography response criteria in solid tumors; PR, partial response; RECIST, response evaluation criteria in solid tumors; mRECIST, modified RECIST; SD, stable disease; WHO, World Health Organization. Asterisks indicate studies using overlapping patient sample. 


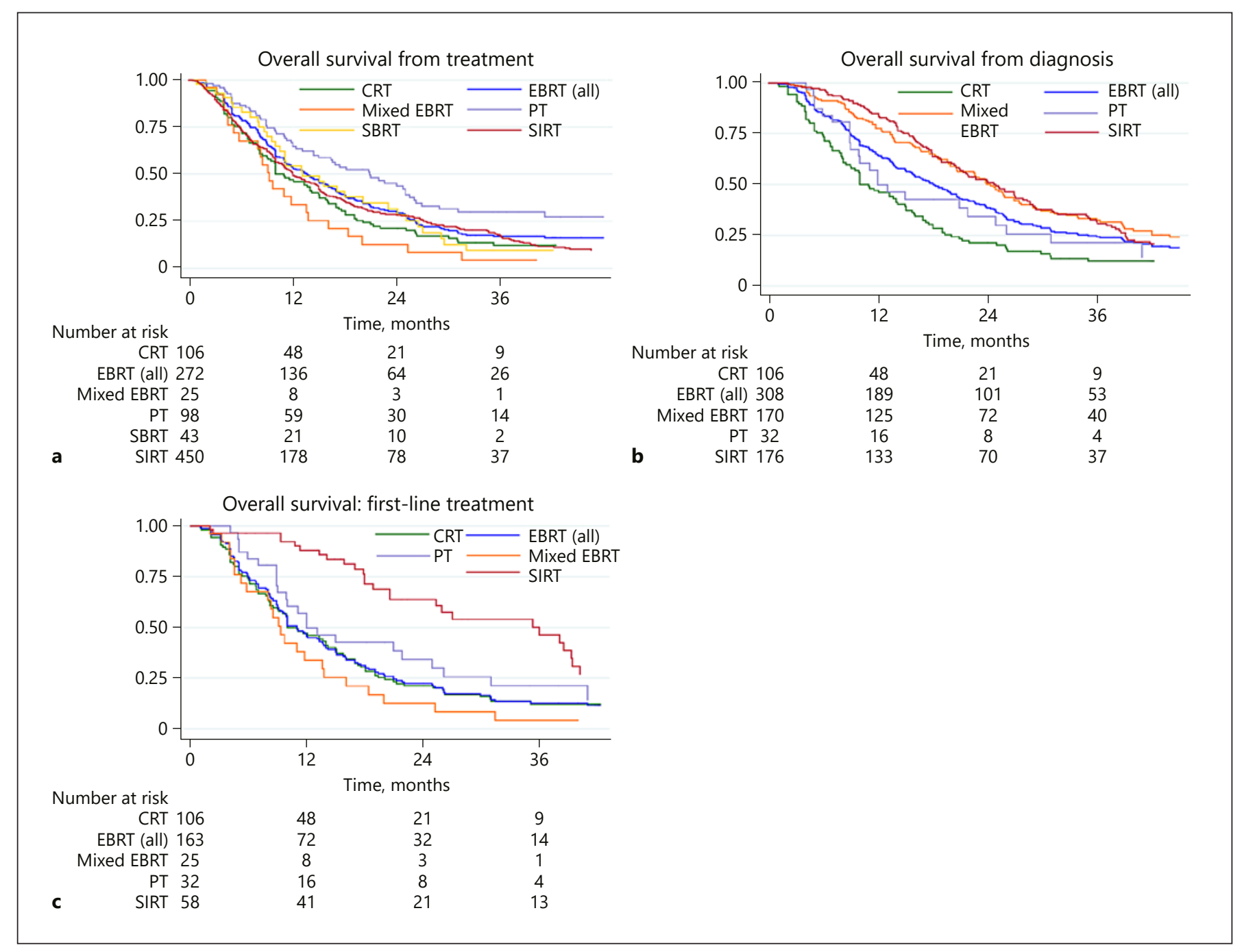

Fig. 2. Kaplan-Meier survival curve of patients who underwent SIRT or EBRT. a Survival from initial treatment. b Survival from initial diagnosis. c Survival among treatment-naive patients who received SIRT or external radiation therapy. CRT, conventional dose radiation therapy (photon); PT, proton beam radiation therapy; SBRT, stereotactic-body radiation therapy; SIRT, selective-internal radiation therapy; EBRT, external beam radiation therapy; OS, overall survival. Mixed: EBRT studies including patients who received more than one type of modality from CRT, SBRT, and PT.

14.4-25) months. The SBRT group had OS rates of $85.4 \%$ (70.3-93.1), 56.1\% (39.7-69.6), 34.2\% (20.1-48.8), and $15.4 \%(6.2-28.3)$ at 6 months, 1 year, 2 years, and 3 years, whereas CRT group had OS rates of 74.3\% (64.8-81.6), $47.4 \%$ (37.6-56.5), 22.1\% (14.7-30.5), and $12.8 \%$ (7.220.0), respectively.

From the time of iCCA diagnosis (Fig. 2b) $[14,24,25$, $35,36,39,41,48,49,51-54,62]$, the MST of SIRT $(n=$ $176)$ and EBRT $(n=308)$ were 25 (95\% CI: 20.6-28.5) versus 18.1 (95\% CI: 9.3-13.6) months, with OS rates of 74.3\% (95\% CI: 64.8-81.6\%), 85.0\% (95\% CI: 78.7-89.6\%),
54.2\% (95\% CI: $46.2-61.6 \%$ ), and $37.0 \%$ (95\% CI: $29.4-$ $44.5 \%)$ at $6,12,24$, and 36 months, respectively, versus 84.3\% (95\% CI: 79.7-87.9\%), 64.9\% (95\% CI: 59.2-70.0\%), $38.2 \%$ (95\% CI: $33.6-44.8 \%$ ), and $25.7 \%$ (95\% CI: $20.8-$ $30.8 \%)$.

Among patients who were treatment-naive $[24,25,49$, $51,53,54,62$ ] (Fig. 2c), the MST for SIRT $(n=58)$ and EBRT $(n=163)$ were 36.1 (95\% CI: 20.6-39.5) and 11.0 (95\% CI: 9.3-13.6) months, respectively. The OS rates of SIRT were 96.4\% (95\% CI: 86.2-99.1\%), 88.6\% (95\% CI: 76.3-94.7\%), 66.3\% (95\% CI: $51.0-77.8 \%$ ), and $56.0 \%$ 
Table 4. Radiographical and survival outcomes of included studies on RT

\begin{tabular}{|c|c|c|c|c|}
\hline Study & Criteria & Radiological outcome & MST, months & OS \\
\hline Shimizu et al. [49]* & RECIST & 3 mo DCR: $36 / 37$ & 15.0 & $\begin{array}{l}6 \text { mo: } 83.6 \% \\
1 \text { yr: } 60.3 \% \\
2 \mathrm{yr}: 41.4 \% \\
3 \mathrm{yr}: 31.0 \%\end{array}$ \\
\hline Ohkawa et al. [50]* & Not shown & Not shown & Not shown & Not shown \\
\hline Shen et al. [13] & mRECIST & $\begin{array}{l}\text { CR: } 3 / 28(10.7 \%) \\
\text { PR: } 10 / 28(35.7 \%) \\
\text { SD: } 12 / 28(42.9 \%) \\
\text { PD: } 3 / 28(10.7 \%)\end{array}$ & 15.0 & $\begin{array}{l}6 \text { mo: } 89.3 \% \\
1 \text { yr: } 57.1 \% \\
2 \text { yr: } 32.1 \\
3 \text { yr: } 10.7 \%\end{array}$ \\
\hline Kim et al. [53] & RECIST & $\begin{array}{l}\text { CR: } 0 \\
\text { PR: } 1 / 25(4.0 \%) \\
\text { SD: } 13 / 25(52.0 \%) \\
\text { PD: } 11 / 25(44.0 \%)\end{array}$ & 9.3 & $\begin{array}{l}6 \text { mo: } 67.7 \% \\
1 \text { yr: } 30.4 \% \\
2 \text { yr: } 12.5 \% \\
3 \text { yr: NA }\end{array}$ \\
\hline Chen et al. [54]** & EASL & $\begin{array}{l}\text { CR: } 3 / 35(8.6 \%) \\
\text { PR: } 10 / 35(28.5 \%) \\
\text { SD: } 17 / 35(48.6 \%) \\
\text { PD: } 5 / 35(14.3 \%)\end{array}$ & 5.4 & $\begin{array}{l}6 \text { mo: } 76.5 \\
1 \text { yr: } 38.5 \% \\
2 \text { yr: } 9.6 \% \\
3 \text { yr\%: NA }\end{array}$ \\
\hline Zeng et al. [55] ${ }^{* *}$ & Not shown & Not shown & Not shown & Not shown \\
\hline Hijazi et al. [57] & RECIST & 3 mo DCR: $3 / 4$ (75\%) & 10 & $\begin{array}{l}6 \text { mo: } 79.9 \% \\
1 \text { yr: } 79.9 \% \\
2 \text { yr: } 0 \\
3 \text { yr: } 0\end{array}$ \\
\hline Ibarra et al. [58] & RECIST & $\begin{array}{l}\text { CR: } 1 / 9(11.1 \%) \\
\text { PR: } 2 / 9(22.2 \%) \\
\text { SD: } 2 / 9(22.2 \%) \\
\text { PD: } 4 / 9(44.4 \%)\end{array}$ & 11 & $\begin{array}{l}6 \text { mo: } 75 \% \\
1 \text { yr: } 45 \% \\
2 \text { yr: NA } \\
3 \text { yr: NA }\end{array}$ \\
\hline Sumiyoshi et al. [62] & RECIST & $\begin{array}{l}\text { CR: } 0 \\
\text { PR: } 4 / 7(57.1 \%) \\
\text { SD: } 1 / 7(14.3 \%) \\
\text { PD: } 2 / 7(28.6 \%)\end{array}$ & Needs calculation & $\begin{array}{l}6 \mathrm{mo}: 80 \% \\
1 \mathrm{yr}: 40 \% \\
2 \mathrm{yr}: 20 \% \\
3 \mathrm{yr}: 20 \%\end{array}$ \\
\hline Kasuya et al. [59] & Not shown & Not shown & 23.8 & $\begin{array}{l}6 \text { mo: } 96.3 \% \\
1 \text { yr: } 77.7 \% \\
2 \text { yr: } 53.2 \% \\
3 \text { yr: } 36.9 \%\end{array}$ \\
\hline Smart et al. $[48]^{* * *}$ & NA & NA & Not shown & $\begin{array}{l}6 \text { mo: } 90.8 \% \\
1 \text { yr: } 79.1 \% \\
2 \text { yr: } 58 \% \\
3 \text { yr: } 30.2 \%\end{array}$ \\
\hline Hong et al. [65] $]^{* * *}$ & RECIST & 3 mo DCR: $36 / 36$ & Not shown & Not shown \\
\hline Yoon et al. [66] $]^{* * *}$ & RECIST & Not shown & Not shown & Not shown \\
\hline Tao et al. [52] $]^{* * *}$ & NA & NA & 30 & $\begin{array}{l}6 \text { mo: } 98.7 \% \\
1 \text { yr: } 87 \% \\
2 \text { yr: } 61 \% \\
3 \text { yr: } 44 \%\end{array}$ \\
\hline Ben-Josef et al. $[56,70]$ & NA & $\begin{array}{l}\text { CR: } 4 / 33(12.1 \%) \\
\text { PR: } 8 / 33(24.2 \%) \\
\text { SD: } 20 / 33(60.6 \%) \\
\text { PD: } 1 / 33(3.0 \%)\end{array}$ & NA & NA \\
\hline
\end{tabular}


Table 4 (continued)

\begin{tabular}{|c|c|c|c|c|}
\hline Study & Criteria & Radiological outcome & MST, months & OS \\
\hline Cho et al. [51] & RECIST & $\begin{array}{l}\text { CR: } 0 \\
\text { PR: } 16 / 56(25 \%) \\
\text { SD: NA } \\
\text { PD: NA }\end{array}$ & 10 & $\begin{array}{l}6 \text { mo: } 67.2 \% \\
1 \text { yr: } 48.4 \% \\
2 \text { yr: } 25 \% \\
3 \text { yr: } 15.8 \%\end{array}$ \\
\hline Kozak et al. [60] & NA & NA & 23 & $\begin{array}{l}6 \text { mo: } 95.5 \% \\
1 \text { yr: } 76.2 \% \\
2 \text { yr: } 47.3 \% \\
3 \text { yr: } 34.1 \%\end{array}$ \\
\hline Liu et al. [61] & RECIST & $\begin{array}{l}\text { CR: } 1 / 12(8.3 \%) \\
\text { PR: } 6 / 12(50.0 \%) \\
\text { SD: } 3 / 12(25.0 \%) \\
\text { PD: } 2 / 12(16.7 \%)\end{array}$ & NA & NA \\
\hline Tse et al. [63] & RECIST & NA & 15 & $\begin{array}{l}6 \text { mo: } 79.6 \% \\
1 \text { yr: } 58.2 \% \\
2 \text { yr: } 28.4 \% \\
3 \text { yr: NA }\end{array}$ \\
\hline Weiner et al. [64] & EASL & $\begin{array}{l}\text { CR: } 1 / 12(8.3 \%) \\
\text { PR: 3/12 (25\%) } \\
\text { SD: NA } \\
\text { PD: NA }\end{array}$ & NA & NA \\
\hline
\end{tabular}

CR, complete response; DCR, disease control rate; EASL, European Association for the Study of the Liver; MST, median survival time; NA, not reported by original study; Not shown, data were not displayed in the table because the outcome was reported by another larger study with overlapping patient sample; OS, overall survival; $\mathrm{PD}$, progressive disease; PERCIST, positron emission tomography response criteria in solid tumors; PR, partial response; RECIST, response evaluation criteria in solid tumors; mRECIST, modified RECIST; SD, stable disease; WHO, World Health Organization. Asterisks indicate studies using overlapping patient sample.

Table 5. Downstaging to surgery

\begin{tabular}{|c|c|c|c|}
\hline Study & Intervention & Surgery & $\begin{array}{l}\text { Treatment-naive } \\
\text { patients who } \\
\text { received surgery }\end{array}$ \\
\hline Buettner et al. [1, 32] & SIRT & $5 / 115(4.3 \%)$ & $1 / 14$ \\
\hline Beuzit et al. [23]* & SIRT & $9 / 45(20 \%)$ & NS \\
\hline Edeline et al. [14]* & SIRT & $11 / 24(46 \%)$ & NS \\
\hline Edeline et al. [24]* & SIRT & $9 / 41(22.0 \%)$ & NS \\
\hline Manceau et al. [21]* & SIRT & $17 / 35(49 \%)$ & $17 / 35$ \\
\hline Rayar et al. [47]* & SIRT & Downstaging-only: 8 pts & NS \\
\hline Riby et al. [46]* & SIRT & Downstaging-only: 19 pts & NS \\
\hline Mouli et al. [33]** & SIRT & $5 / 46(11 \%)$ & $5 / 18$ \\
\hline Ibrahim et al. [34] $]^{* *}$ & SIRT & $2 / 24(8.3 \%)$ & NS \\
\hline Saxena et al. [37] & SIRT & $1 / 25(4 \%)$ & $1 / 6$ \\
\hline Shaker et al. [45] & SIRT & $1 / 17(5.9 \%)$ & $1 / 9$ \\
\hline Cho et al. [51] & EBRT & $8 / 64(12.5 \%)$ & $8 / 64$ \\
\hline Sumiyoshi et al. [62] & EBRT & $5 / 7(71.4 \%)$ & $5 / 7$ \\
\hline
\end{tabular}

SIRT, selective-internal radiation therapy; EBRT, external body radiation therapy; NS, not shown due to overlapping patients. Asterisks indicate studies using overlapping patient sample. 
Table 6. Pooled post-procedural complications after SIRT or EBRT

\begin{tabular}{lll}
\hline Complication & SIRT & EBRT \\
\hline Ulcer & $2 / 198(1.0 \%)$ & $2 / 388(0.5 \%)$ \\
Nausea & $3 / 190(1.6 \%)$ & $2 / 276(0.7 \%)$ \\
Vomiting & $1 / 181(0.6 \%)$ & $2 / 263(0.7 \%)$ \\
Anorexia & $14 / 238(5.9 \%)$ & $0 / 24(0 \%)$ \\
Abdominal pain & $9 / 130(6.9 \%)$ & $11 / 492(2.2 \%)$ \\
Anemia & $1 / 154(0.6 \%)$ & $8 / 105(7.5 \%)$ \\
Neutropenia & $10 / 154(6.5 \%)$ & $21 / 191(11.0 \%)$ \\
Thrombocytopenia & $12 / 164(7.3 \%)$ & $10 / 167(6.0 \%)$ \\
ALP & $2 / 127(1.6 \%)$ & $2 / 238(0.8 \%)$ \\
AST & $1 / 147(0.7 \%)$ & $3 / 297(1.0 \%)$ \\
ALT & $3 / 147(2.0 \%)$ & $0 / 288(0 \%)$ \\
Bilirubin & $9 / 174(5.2 \%)$ & $8 / 381(2.1 \%)$ \\
Albumin & $5 / 38(13.2 \%)$ & $5 / 151(3.3 \%)$ \\
\hline
\end{tabular}

SIRT, selective-internal radiation therapy; EBRT, external body radiation therapy.

(95\% CI: 40.5-69.0) at 6 months, 1 year, 2 years, and 3 years, respectively, compared with those of EBRT: $74.7 \%$ (95\% CI: $67.3-80.7 \%$ ), $47.0 \%$ (95\% CI: $39.2-54.5 \%$ ), 23.3\% (95\% CI: $17.0-30.1 \%$ ), and $13.7 \%$ (95\% CI: $8.9-$ $19.6 \%)$. By subgroups of EBRT, the MST of PT $(n=32)$, and CRT $(n=106)$ were 12.0 (95\% CI: 9.0-25.0) and 10.0 (95\% CI: 8.5-14.2) months, respectively. No survival data regarding SBRT as the first-line treatment could be pooled.

\section{Downstaging to Surgery}

Nine studies of SIRT reported the number of initially unresectable iCCA that were eventually resected after neoadjuvant therapy (Table 5). The proportion of patients who eventually received surgery ranged from 4 to $49 \%$, excluding studies that solely focused on downstaged patients. One patient was downstaged to transplant [34]. By contrast, 12.5-71.4\% patients who underwent EBRT were downstaged to surgical resection; these patients received conventional dose 3 DCRT or IMRT. Among patients who were treatment-naive, a cumulative of $30.5 \%$ $(25 / 82)$ and $18.3 \%(13 / 71)$ of patients who received SIRT and EBRT were downstaged to surgery. Patient-level survival data were available in 26 patients (Fig. 3). Neither group reached MST, with a 1-year OS of $90.1 \%$ (95\% CI: 67.7-97.6\%) and 51.5\% (95\% CI: 30.8-81.8\%) in SIRT and EBRT groups, respectively.

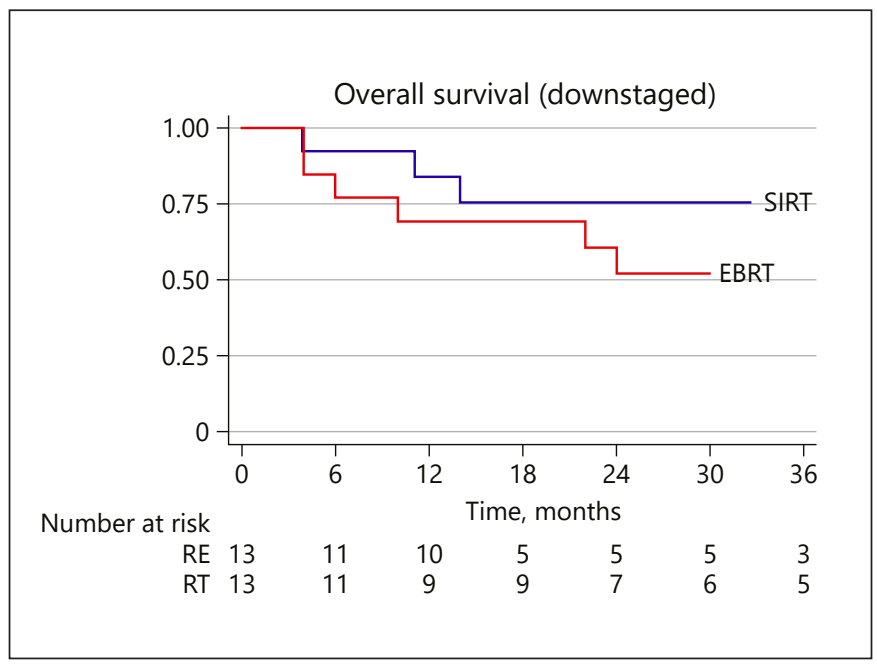

Fig. 3. OS rates among patients who were downstaged to surgery after SIRT or EBRT. SIRT, selective-internal radiation therapy; EBRT, external beam radiation therapy; OS, overall survival.

\section{Toxicity}

The pooled severe complications are listed in Table 6, with the specific event from each study listed in online suppl. Tables 3.1 and 3.2. Compared to the SIRT group, patients who underwent EBRT had a higher rate of severe complications including: peptic ulcer ( 1 vs. $0.5 \%)$, nausea (1.6 vs. $0.7 \%$ ), anorexia ( $6.0 \%$ vs. 0$)$, abdominal pain (6.9 vs. $2.2 \%$ ), thrombocytopenia (7.3 vs. $6.0 \%$ ), hypoalbuminemia (13.2 vs. $3.3 \%)$, and elevations in liver enzymes - ALP (1.6 vs. $0.8 \%$ ), ALT ( $2 \%$ vs. 0 ), and bilirubin (5.2 vs. $2.1 \%)$. SIRT had a higher rate of major complication in anemia (7.5 vs. $0.6 \%)$, neutropenia ( 11.0 vs. $6.5 \%)$, and AST elevation (1.0\% vs. 0.7). Radiation-induced dermatitis only occurred in EBRT patients $[48,49,53]$, with a frequency from 28 to $88 \%$, but not the SIRT patients. No radiation-induced pneumonitis was observed.

\section{Discussion}

Both SIRT and EBRT are acceptable treatments for unresectable iCCA in a variety of clinical scenarios including first-line therapy, chemorefractory tumors, recurrence of cancer, as combination therapy (in conjunction with chemotherapy), or neoadjuvant therapy $[1,11]$. A head-to-head comparison of EBRT versus SIRT under each specific clinical setting remains difficult, as most published literature on this topic includes a widely heterogeneous patient population. Limited evidence is avail- 
able regarding the comparative efficacy of SIRT versus EBRT in treating liver malignancies. Based on the National Cancer Database, Oladeru et al. [67] indicated that $\mathrm{RE}$ and SBRT resulted in similar survival benefits in patients with unresectable HCC, whereas Sebastian et al. [17] suggested that the latter was superior to both SIRT and conventional dose chemoradiotherapy in cholangiocarcinoma. In a meta-analysis of 37 studies on HCC with PVI, OS rates among 3D CRT, SIRT, and SBRT were comparable [68]. None of the available evidence including the studies mentioned above focused on SIRT versus EBRT as the first-line treatment, which might otherwise be prone to selection bias. Likewise, in the present study, the prespecified primary end point of OS between treatment arms proved to be difficult due to different baseline conditions among the SIRT and EBRT groups, as the former was often implemented as salvage rather than primary treatment (Tables 1,2). To account for this, the present study reported OS of treatment-naive patients, in addition to from the time of radiation treatment and iCCA diagnosis. Among treatment-naive patients (Fig. 2e, f), the MST for SIRT and EBRT were 36.1 (95\% CI: 20.639.5) and 11.0 (95\% CI: 9.3-13.6) months, respectively; the MST of PT and CRT were 12.0 (95\% CI: 9.0-25.0) and 10.0 (95\% CI: 8.5-14.2) months, respectively. Though the survival of SBRT among treatment-naive patients was unknown, the OS from initial SBRT was 13.1 (95\% CI: 10.0-21.1) months. While the baseline heterogeneity between 2 groups prevents high-quality comparative analysis, the present study offers baseline survival outcomes for future RCTs comparing these 2 treatment modalities.

For advanced iCCA, the National Comprehensive Cancer Network guideline recommends cisplatin-gemcitabine chemotherapy as first-line treatment [69]. Among included studies of the present meta-analysis, $60.1 \%$ (514/855) of SIRT and 29.7\% (135/454) EBRT patients received prior chemotherapy, while the percentage of patients who received concomitant chemotherapy were $43.4 \%(151 / 348)$ and $55.6 \%(175 / 315)$, respectively (Tables 1,2$)$. Among treatment-naive subgroup analysis, $60.3 \%(35 / 58)$ and $68.7 \%(112 / 163)$ patients received SIRT and EBRT combined with chemotherapy (Tables 1, 2). Lamarca et al. [85] performed a post hoc analysis of 66 iCCA patients who were treated with first-line cisplatin-gemcitabine chemotherapy in Advanced Biliary Tract Cancer-01, -02, and -03 studies. Among 51.5\% (34/66) patients with the liver-only disease, the MST was 16.7 (95\% CI: 8.7-22.2) months, compared with the 2 recently published prospective multicenter clinical trials from the present meta-analysis, reporting MST of 22 and 22.5 months for iCCA patients who received SIRT and highdose hypofractionated PT, respectively. Patients with localized disease burden demonstrated longer OS than those with extrahepatic metastasis, which did not reach statistical significance $(\mathrm{HR}=0.65, p=0.16)$ due to underpowering. The addition of locoregional therapy, such as EBRT and SIRT on top of chemotherapy could potentially be beneficial in this patient group if surgery were contraindicated.

The baseline heterogeneity between SIRT and EBRT groups was difficult to adjust due to the nature of studylevel data. Reported predictors of survival among iCCA patients receiving radiation therapy include lesion number [33], lobar distribution [42], history of surgery [42], BED [52], adjuvant chemotherapy administration [49], and the presence of jaundice [49]. These variables can serve as a reference for future RCT design and stratification. One additional difficulty in comparing EBRT versus SIRT is standardizing effective radiation doses between 2 modalities. A higher radiation dose correlates positively with the survival [70]. Theoretically, SIRT can deliver higher concentrated radiation doses to tumor than EBRT with a sustained radiation for approximately 2 weeks post-procedurally [71]. While SIRT studies often report in GBy, EBRT reports total delivered doses and BEDs (Tables 1, 2). BED of SIRT has been calculated with the consideration of time factors, despite downsides such as single-photon emission computed tomography being less accurate than PET/CT $[72,73]$. The development of a conversion algorithm may be needed to characterize the relationship of radiation dosage and clinical outcomes in future studies comparing EBRT and SIRT.

A variety of imaging criteria were adopted to evaluate posttreatment objective radiographical response in the present meta-analysis: RECIST, mRECIST, PERCIST, WHO, and EASL (Fig. 1). Most included studies measured radiologic response according to the RECIST criteria. The RR and DCR seemed to be higher in patients who received EBRT than SIRT: $22 \%$ (95\% CI: $10-37 \%$ ) versus $31 \%$ (95\% CI: $12-54 \%$ ) and 75\% (95\% CI: 65-84\%) versus 83\% (95\% CI: 61-98\%). Yet, RECIST has a questionable reliability in the evaluation of locoregional therapy (including RE) responses in hepatic malignancies [74-77]. Because Y-90 RE induced tumor necrosis often accompanied peripheral edema, the objective radiographical response could be underestimated and may not necessarily correlate with survival [78]. Camacho et al. [39] retrospectively evaluated 9 patients treated with Y-90 radioembolization and showed that RECIST poorly correlated with OS. Likewise, in a retrospective cohort of 45 patients who 
received SIRT for iCCA, Beuzit et al. [23] did not observe significant survival difference based on RECIST, but Choi responders correlated with prolonged survival difference. Few studies implemented the PERCIST criteria, which provide a more accurate assessment of tumor metabolic activity than RECIST $[25,30]$. Further, the posttreatment imaging follow-up intervals varied among studies. While most studies measured the objective radiological $R R$ at 3 -month post embolization [23,31,41], some only reported these outcomes at 1-month follow-up or the best responses achieved $[28,51,56]$. As such, standardization of posttreatment objective radiological response evaluation is needed between interventional radiologists and radiation oncologists to allow future comparative analysis.

As the definitive treatment of iCCA, surgical resection is determined by an interplay of tumor number, vascular/ ductal invasion, the presence of extrahepatic disease, and hepatic functional reserve [79-82]. The present study suggested that both SIRT and EBRT effectively reduced tumor burden and could downstage initially unresectable tumors to surgery (Fig. 3), despite small sizes ( $n=13$ in each group). Of note, both SIRT and EBRT could only be implemented to bridge surgery when tumor size was the limiting factor. Among the 8 patients who were downstaged to surgery in the study of Rayar et al. [47], the initial unresectability was due to tumoral involvement of the hepatic vein and portal vein. In the study by Mouli et al. [33], all 13 initially "downstageable" cases were solitary lesions without cirrhosis, and the 5 downstaged patients were ECOG 0 without portal vascular invasion. Further, in the 9 patients of Edeline et al. [24], tumor regression was achieved in tumors that initially invaded hepatic and portal veins, allowing surgical hepatectomy. Interestingly, it was also observed that the liver remnant volume also increased after SIRT, suggesting that SIRT promoted liver hypertrophy and enhanced hepatic function reserve [47], which may broaden patient's surgical candidacy and warrant validation from future studies. As for EBRT, in the study of Sumiyoshi et al. [62], the reasons of unresectability included 4 extrahepatic perineural invasions and 3 vascular invasions to hepatic vein and IVC. Tumor size reduction was observed in all cases; 5 eventually received surgery, while 2 developed extrahepatic metastases and thus lost candidacy. Among the 8 downstaged patients in the study of Cho et al. [51], 3 presented with major vessel invasion; tumor regression was observed in all cases after EBRT. Despite the pooled successful downstage rate among treatment-naive patients being lower in the SIRT group (30.5 vs. $18.3 \%$ ), such analysis should be interpreted with caution because the proportion of patients who were "downstageable" was unknown. For example, patients who

iCCA: Radioembolization versus External Radiation Therapy presented with extrahepatic metastases, had poor baseline neurofunctional status, and received treatment with a palliative intent should not be considered in the baseline treatment group in such analyses. This is particularly relevant for EBRT, where only patients receiving curative radiation dosages should be considered. Thus, whether SIRT is truly superior to EBRT in downstaging iCCA remains to be answered by high-quality comparative studies.

The overall toxicity profile from included studies was low. Clinical complications, such as severe peptic ulcer disease, nausea, anorexia, and abdominal pain occurred more frequently in the EBRT cohort (Table 6). In addition, radiation-related dermatitis was exclusive to EBRT. All neutropenia, anemia, and thrombocytopenia cases among the SIRT group occurred in 1 study that combined SIRT with chemotherapy, suggesting enhanced bone marrow-related toxicity when these 2 modalities were administered simultaneously $[24,83]$. Two cases of radiation-induced liver disease (RILD) occurred in the EBRT arm: one case resolved with corticosteroids and the other resulted in death $[48,54]$. In comparison, $2 / 5$ RILD cases in the SIRT group were considered life-threatening, though specific details were not provided by the original study $[32,38]$. While PT should reduce complications observed with photon-based RT owing to Bragg peak [84], subgroup analyses based on RT subtypes were not possible for the rare incidence in the present study.

The present meta-analysis should be interpreted with several caveats. First, each treatment arm was pooled independently from single-arm studies. Because of the variations in data reporting, the baseline characteristics of each treatment group could not be pooled and compared. For example, technical variances among institutions and additional patient-specific characteristic might also contribute to underlying heterogeneity. Such heterogeneity prohibits a head-to-head comparison between these 2 treatment modalities. Second, although individual survival data was retrieved from original studies, several studies did not label "tick" (censored data). In addition, some studies had overlapping ticks, and therefore not every patient could be mapped. The decision to exclude these studies inevitably led to the loss of a large proportion of available data. However, excluding censored data would underestimate the true survival rate. Third, stratification based on curative versus palliative intent was not performed, as the original studies rarely provided outcomes with such categorizations. Finally, many EBRT studies including iCCA patients were excluded as these studies also included hilar and distal CCA, which could certainly lead to the present study being underpowered. 
Robust evidence has demonstrated that the genetics, molecular progression, and clinical behaviors of these 3 subgroups of CCA are distinct from one another and therefore should be treated as distinct entities.

\section{Conclusion}

The present study reviewed the available evidence of EBRT and SIRT in the management of unresectable iCCA. Both treatments demonstrated efficacy with acceptable toxicity profiles and can serve as bridging treatment to surgery. Survival benefits varied among different subtypes of EBRT. There was no consensus regarding the most reliable radiographic criteria comparing the treatment efficacies of SIRT and EBRT. Patients with iCCA who underwent SIRT versus EBRT were heterogeneous in treatment approach and disease severity which limited the ability to make broad conclusions. As such, given the noncomparative nature of each included study, future prospective, multicenter randomized controlled trials are needed to determine superiority of specific radiotherapies in iCCA.

\section{Statement of Ethics}

The authors have no ethical conflicts to disclose. The research was conducted ethically in accordance with the World Medical Association Declaration of Helsinki. Ethical review and approval was not required as the study is based exclusively on published literature.

\section{Conflict of Interest Statement}

The authors have no conflict of interest to declare.

\section{Funding Sources}

The authors did not receive any funding.

\section{Author Contributions}

Q.Y.: data extraction, manuscript writing, and data analysis. C.L.: revision and manuscript writing. A.P.: manuscript writing. O.A.: data extraction, manuscript writing, and data analysis.

\section{References}

1 Buettner S, van Vugt JL, IJzermans JN, Groot Koerkamp B. Intrahepatic cholangiocarcinoma: current perspectives. Onco Targets Ther. 2017;10:1131.

2 Taylor-Robinson S, Toledano M, Arora S, Keegan T, Hargreaves S, Beck A, et al. Increase in mortality rates from intrahepatic cholangiocarcinoma in England and Wales 1968-1998. Gut. 2001;48(6):816-20.

3 Patel T. Increasing incidence and mortality of primary intrahepatic cholangiocarcinoma in the United States. Hepatology. 2001;33(6):1353-7.

4 Patel T. Worldwide trends in mortality from biliary tract malignancies. BMC cancer. 2002; 2(1): 10 .

5 Farges O, Fuks D. Clinical presentation and management of intrahepatic cholangiocarcinoma. Gastroenterol Clin Biol. 2010;34(3): 191-9.

6 Tan JC, Coburn NG, Baxter NN, Kiss A, Law $\mathrm{CH}$. Surgical management of intrahepatic cholangiocarcinoma: a population-based study. Ann Surg Oncol. 2008;15(2):600-8.

7 Wang Y, Li J, Xia Y, Gong R, Wang K, Yan Z, et al. Prognostic nomogram for intrahepatic cholangiocarcinoma after partial hepatectomy. J Clin Oncol. 2013;31(9):1188-95.

8 Ribero D, Pinna AD, Guglielmi A, Ponti A, Nuzzo G, Giulini SM, et al. Surgical approach for long-term survival of patients with intrahepatic cholangiocarcinoma: a multi-institutional analysis of 434 patients. Arch Surg. 2012;147(12):1107-13.
9 Farges O, Fuks D, Boleslawski E, Le Treut YP, Castaing D, Laurent A, et al. Influence of surgical margins on outcome in patients with intrahepatic cholangiocarcinoma: a multicenter study by the AFC-IHCC-2009 study group. Ann Surg. 2011;254(5):824-30.

10 Morimoto Y, Tanaka Y, Ito T, Nakahara M, Nakaba H, Nishida T, et al. Long-term survival and prognostic factors in the surgical treatment for intrahepatic cholangiocarcinoma. J Hepatobiliary Pancreat Surg. 2003;10(6):432-40.

11 Bridgewater J, Galle PR, Khan SA, Llovet JM, Park JW, Patel T, et al. Guidelines for the diagnosis and management of intrahepatic cholangiocarcinoma. J Hepatol. 2014;60(6): 1268-89.

12 Jackson MW, Amini A, Jones BL, Rusthoven CG, Schefter TE, Goodman KA. Treatment selection and survival outcomes with and without radiation for unresectable, localized intrahepatic cholangiocarcinoma. Cancer J. 2016;22(4):237-42.

13 Shen ZT, Zhou H, Li AM, Li B, Shen JS, Zhu XX. Clinical outcomes and prognostic factors of stereotactic body radiation therapy for intrahepatic cholangiocarcinoma. Oncotarget. 2017;8(55):93541-50.

14 Edeline J, Du FL, Rayar M, Rolland Y, Beuzit L, Boudjema K, et al. Glass microspheres 90Y selective internal radiation therapy and chemotherapy as first-line treatment of intrahepatic cholangiocarcinoma. Clin Nucl Med. 2015;40(11):851-5.
15 Al-Adra DP, Gill RS, Axford SJ, Shi X, Kneteman N, Liau SS. Treatment of unresectable intrahepatic cholangiocarcinoma with yttrium-90 radioembolization: a systematic review and pooled analysis. Eur J Surg Oncol. 2015;41(1):120-7.

16 Benson AB, D'Angelica MI, Abbott DE, Abrams TA, Alberts SR, Saenz DA, et al. NCCN guidelines insights: hepatobiliary cancers, version 1.2017. J Natl Compr Canc Netw. 2017;15(5):563-73.

17 Sebastian NT, Tan Y, Miller ED, Williams TM, Alexandra Diaz D. Stereotactic body radiation therapy is associated with improved overall survival compared to chemoradiation or radioembolization in the treatment of unresectable intrahepatic cholangiocarcinoma. Clin Transl Radiat Oncol. 2019;19:66-71.

18 Nyaga V, Arbyn M, Aerts M. METAPROP Stata module to perform fixed and random effects meta-analysis of proportions; 2017.

19 Harris RJ, Deeks JJ, Altman DG, Bradburn MJ, Harbord RM, Sterne JA. Metan: fixedand random-effects meta-analysis. Stata J. 2008;8(1):3-28.

20 Akinwande O, Shah V, Mills A, Noda C, Weiner E, Foltz G, et al. Chemoembolization versus radioembolization for the treatment of unresectable intrahepatic cholangiocarcinoma in a single institution image-based efficacy and comparative toxicity. Hepat Oncol. 2017; $4(3): 75-81$ 
21 Manceau V, Palard X, Rolland Y, Pracht M, Le Sourd S, Laffont S, et al. A MAA-based dosimetric study in patients with intrahepatic cholangiocarcinoma treated with a combination of chemotherapy and (90)Y-loaded glass microsphere selective internal radiation therapy. Eur J Nucl Med Mol Imaging. 2018; 45(10):1731-41.

22 Rafi S, Piduru SM, El-Rayes B, Kauh JS, Kooby DA, Sarmiento JM, et al. Yttrium-90 radioembolization for unresectable standard-chemorefractory intrahepatic cholangiocarcinoma: survival, efficacy, and safety study. Cardiovasc Intervent Radiol. 2013;36(2):4408.

23 Beuzit L, Edeline J, Brun V, Ronot $\mathrm{M}$, Guillygomarc'h A, Boudjema K, et al. Comparison of Choi criteria and Response Evaluation Criteria in Solid Tumors (RECIST) for intrahepatic cholangiocarcinoma treated with glass-microspheres Yttrium-90 selective internal radiation therapy (SIRT). Eur J Radiol. 2016;85(8):1445-52.

24 Edeline J, Touchefeu Y, Guiu B, Farge O, Tougeron D, Baumgaertner I, et al. Radioembolization plus chemotherapy for first-line treatment of locally advanced intrahepatic cholangiocarcinoma: a phase 2 clinical trial. JAMA Oncol. 2019;6(1):51-9.

25 Filippi L, Pelle G, Cianni R, Scopinaro F, Bagni O. Change in total lesion glycolysis and clinical outcome after (90)Y radioembolization in intrahepatic cholangiocarcinoma. Nucl Med Biol. 2015;42(1):59-64.

26 Filippi L, Di Costanzo GG, Tortora R, Pelle G, Saltarelli A, Marino Marsilia G, et al. Prognostic value of neutrophil-to-lymphocyte ratio and its correlation with fluorine-18-fluorodeoxyglucose metabolic parameters in intrahepatic cholangiocarcinoma submitted to $90 \mathrm{Y}$ radioembolization. Nucl Med Commun. 2020;41(1):78-86.

27 Filippi L, Di Costanzo GG, Tortora R, Pelle G, Cianni R, Schillaci O, et al. Repeated treatment with (90)Y-microspheres in intrahepatic cholangiocarcinoma relapsed after the first radioembolization. Cancer Biother Radiopharm. 2019;34(4):231-7.

28 Mosconi C, Cucchetti A, Bruno A, Cappelli A, Bargellini I, De Benedittis C, et al. Radiomics of cholangiocarcinoma on pretreatment CT can identify patients who would best respond to radioembolisation. Eur Radiol. 2020;30(8): 4534-44.

29 Mosconi C, Gramenzi A, Ascanio S, Cappelli A, Renzulli M, Pettinato C, et al. Yttrium-90 radioembolization for unresectable/recurrent intrahepatic cholangiocarcinoma: a survival, efficacy and safety study. Br J Cancer. 2016; 115(3):297-302.

30 Haug AR, Heinemann V, Bruns CJ, Hoffmann R, Jakobs T, Bartenstein P, et al. 18FFDG PET independently predicts survival in patients with cholangiocellular carcinoma treated with $90 \mathrm{Y}$ microspheres. Eur J Nucl Med Mol Imaging. 2011;38(6):1037-45.
31 Hoffmann RT, Paprottka PM, Schön A, Bamberg F, Haug A, Dürr EM, et al. Transarterial hepatic yttrium-90 radioembolization in patients with unresectable intrahepatic cholangiocarcinoma: factors associated with prolonged survival. Cardiovasc Intervent Radiol. 2012;35(1):105-16.

32 Buettner S, Braat AJ, Margonis GA, Brown $\mathrm{DB}$, Taylor KB, Borgmann AJ, et al. Yttrium-90 radioembolization in intrahepatic cholangiocarcinoma: a multicenter retrospective analysis. J Vasc Interv Radiol. 2020; 31(7):1035-43.e2.

33 Mouli S, Memon K, Baker T, Benson AB 3rd, Mulcahy MF, Gupta R, et al. Yttrium-90 radioembolization for intrahepatic cholangiocarcinoma: safety, response, and survival analysis. J Vasc Interv Radiol. 2013;24(8):1227-34.

34 Ibrahim SM, Mulcahy MF, Lewandowski RJ, Sato KT, Ryu RK, Masterson EJ, et al. Treatment of unresectable cholangiocarcinoma using yttrium-90 microspheres: results from a pilot study. Cancer. 2008;113(8):2119-28.

35 Jia Z, Paz-Fumagalli R, Frey G, Sella DM, McKinney JM, Wang W. Resin-based Yttrium-90 microspheres for unresectable and failed first-line chemotherapy intrahepatic cholangiocarcinoma: preliminary results. J Cancer Res Clin Oncol. 2017;143(3):481-9.

36 Reimer P, Virarkar MK, Binnenhei M, Justinger M, Schon MR, Tatsch K. Prognostic factors in overall survival of patients with unresectable intrahepatic cholangiocarcinoma treated by means of Yttrium- 90 radioembolization: results in therapy-naive patients. Cardiovasc Intervent Radiol. 2018;41(5):744-52.

37 Saxena A, Bester L, Chua TC, Chu FC, Morris DL. Yttrium-90 radiotherapy for unresectable intrahepatic cholangiocarcinoma: a preliminary assessment of this novel treatment option. Ann Surg Oncol. 2010;17(2):484-91.

38 White J, Carolan-Rees G, Dale M, Patrick HE, See TC, Bell JK, et al. Yttrium-90 transarterial radioembolization for chemotherapy-refractory intrahepatic cholangiocarcinoma: a prospective, observational study. J Vasc Interv Radiol. 2019;30(8):1185-92.

39 Camacho JC, Kokabi N, Xing M, Prajapati HJ, El-Rayes B, Kim HS. Modified response evaluation criteria in solid tumors and European Association for The Study of the Liver criteria using delayed-phase imaging at an early time point predict survival in patients with unresectable intrahepatic cholangiocarcinoma following yttrium-90 radioembolization. J Vasc Interv Radiol. 2014;25(2):256-65.

40 Xing M, Lahti S, Kokabi N, Schuster DM, Camacho JC, Kim HS. 90 Y radioembolization lung shunt fraction in primary and metastatic liver cancer as a biomarker for survival. Clin Nucl Med. 2016;41(1):21-7.

41 Gangi A, Shah J, Hatfield N, Smith J, Sweeney J, Choi J, et al. Intrahepatic cholangiocarcinoma treated with transarterial Yttrium-90 glass microsphere radioembolization: results of a single institution retrospective study. J Vasc Interv Radiol. 2018;29(8):1101-8.
42 Kohler M, Harders F, Lohofer F, Paprottka PM, Schaarschmidt BM, Theysohn J, et al. Prognostic factors for overall survival in advanced intrahepatic cholangiocarcinoma treated with Yttrium-90 radioembolization. J Clin Med. 2019;9(1):56.

43 Levillain H, Duran Derijckere I, Ameye L, Guiot T, Braat A, Meyer C, et al. Personalised radioembolization improves outcomes in refractory intra-hepatic cholangiocarcinoma: a multicenter study. Eur J Nucl Med Mol Imaging. 2019;46(11):2270-9.

44 Soydal C, Kucuk ON, Bilgic S, Ibis E. Radioembolization with (90)Y resin microspheres for intrahepatic cholangiocellular carcinoma: prognostic factors. Ann Nucl Med. 2016; 30(1):29-34.

45 Shaker TM, Chung C, Varma MK, Doherty MG, Wolf AM, Chung MH, et al. Is there a role for ytrrium-90 in the treatment of unresectable and metastatic intrahepatic cholangiocarcinoma? Am J Surg. 2018;215(3):46770 .

46 Riby D, Mazzotta A, Bergeat D, Verdure L, Sulpice L, Bourien H, et al. Downstaging with radioembolization or chemotherapy for initially unresectable intrahepatic cholangiocarcinoma. Ann Surg Oncol. 2020;27(10):372937.

47 Rayar M, Sulpice L, Edeline J, Garin E, Levi Sandri GB, Meunier B, et al. Intra-arterial yttrium-90 radioembolization combined with systemic chemotherapy is a promising method for downstaging unresectable huge intrahepatic cholangiocarcinoma to surgical treatment. Ann Surg Oncol. 2015;22(9):3102-8.

48 Smart AC, Goyal L, Horick N, Petkovska N, Zhu AX, Ferrone CR, et al. Hypofractionated radiation therapy for unresectable/locally recurrent intrahepatic cholangiocarcinoma. Ann Surg Oncol. 2020;27(4):1122-9.

49 Shimizu S, Okumura T, Oshiro Y, Fukumitsu $\mathrm{N}$, Fukuda K, Ishige K, et al. Clinical outcomes of previously untreated patients with unresectable intrahepatic cholangiocarcinoma following proton beam therapy. Radiat Oncol. 2019;14(1):241

50 Ohkawa A, Mizumoto M, Ishikawa H, Abei M, Fukuda K, Hashimoto T, et al. Proton beam therapy for unresectable intrahepatic cholangiocarcinoma. J Gastroenterol Hepatol. 2015;30(5):957-63.

51 Cho Y, Kim TH, Seong J. Improved oncologic outcome with chemoradiotherapy followed by surgery in unresectable intrahepatic cholangiocarcinoma. Strahlenther Onkol. 2017; 193(8):620-9.

52 Tao R, Krishnan S, Bhosale PR, Javle MM, Aloia TA, Shroff RT, et al. Ablative radiotherapy doses lead to a substantial prolongation of survival in patients with inoperable intrahepatic cholangiocarcinoma: a retrospective dose response analysis. J Clin Oncol. 2016; 34(3):219-26. 
53 Kim YI, Park JW, Kim BH, Woo SM, Kim TH, $\mathrm{Koh} \mathrm{YH}$, et al. Outcomes of concurrent chemoradiotherapy versus chemotherapy alone for advanced-stage unresectable intrahepatic cholangiocarcinoma. Radiat Oncol. 2013;8:292.

54 Chen YX, Zeng ZC, Tang ZY, Fan J, Zhou J, Jiang $\mathrm{W}$, et al. Determining the role of external beam radiotherapy in unresectable intrahepatic cholangiocarcinoma: a retrospective analysis of 84 patients. BMC Cancer. 2010;10:492.

55 Zeng ZC, Tang ZY, Fan J, Zhou J, Qin LX, Ye $\mathrm{SL}$, et al. Consideration of the role of radiotherapy for unresectable intrahepatic cholangiocarcinoma: a retrospective analysis of 75 patients. Cancer J. 2006;12(2):113-22.

56 Ben-Josef E, Normolle D, Ensminger WD, Walker S, Tatro D, Ten Haken RK, et al. Phase II trial of high-dose conformal radiation therapy with concurrent hepatic artery floxuridine for unresectable intrahepatic malignancies. J Clin Oncol. 2005;23(34):8739-47.

57 Hijazi H, Campeau MP, Roberge D, Donath D, Lapointe R, Vandenbroucke-Menu F, et al. Stereotactic body radiotherapy for inoperable liver tumors: results of a single institutional experience. Cureus. 2016;8(12):e935.

58 Ibarra RA, Rojas D, Snyder L, Yao M, Fabien J, Milano M, et al. Multicenter results of stereotactic body radiotherapy (SBRT) for nonresectable primary liver tumors. Acta Oncol. 2012;51(5):575-83.

59 Kasuya G, Terashima K, Shibuya K, Toyama $\mathrm{S}$, Ebner DK, Tsuji H, et al. Carbon-ion radiotherapy for cholangiocarcinoma: a multi-institutional study by and the Japan carbon-ion radiation oncology study group (J-CROS). Oncotarget. 2019;10(43):4369-79.

60 Kozak MM, Toesca DAS, von Eyben R, Pollom EL, Chang DT. Stereotactic body radiation therapy for cholangiocarcinoma: optimizing locoregional control with elective nodal irradiation. Adv Radiat Oncol. 2020; 5(1):77-84.

61 Liu MY, Lo CH, Lin CS, Chao HL, Yang JF, Lin KT, et al. Stereotactic ablative radiotherapy for patients with unresectable or medically inoperable cholangiocarcinoma. Tumori. 2017;103(3):236-41.

62 Sumiyoshi T, Shima Y, Okabayashi T, Negoro Y, Shimada Y, Iwata J, et al. Chemoradiotherapy for initially unresectable locally advanced cholangiocarcinoma. World J Surg. 2018; 42(9):2910-8.

63 Tse RV, Hawkins M, Lockwood G, Kim JJ, Cummings B, Knox J, et al. Phase I study of individualized stereotactic body radiotherapy for hepatocellular carcinoma and intrahepatic cholangiocarcinoma. J Clin Oncol. 2008; 26(4):657-64.

64 Weiner AA, Olsen J, Ma D, Dyk P, DeWees T, Myerson RJ, et al. Stereotactic body radio- therapy for primary hepatic malignancies: report of a phase I/II institutional study. Radiother Oncol. 2016;121(1):79-85.

65 Hong TS, Wo JY, Yeap BY, Ben-Josef E, McDonnell EI, Blaszkowsky LS, et al. Multi-institutional phase II study of high-dose hypofractionated proton beam therapy in patients with localized, unresectable hepatocellular carcinoma and intrahepatic cholangiocarcinoma. J Clin Oncol. 2016;34(5):460-8.

66 Yoon SS, Aloia TA, Haynes AB, Kambadakone A, Kaur H, Vauthey JN, et al. Surgical placement of biologic mesh spacers to displace bowel away from unresectable liver tumors followed by delivery of dose-intense radiation therapy. Pract Radiat Oncol. 2014; 4(3):167-73.

67 Oladeru OT, Miccio JA, Yang J, Xue Y, Ryu S, Stessin AM. Conformal external beam radiation or selective internal radiation therapy: a comparison of treatment outcomes for hepatocellular carcinoma. J Gastrointest Oncol. 2016;7(3):433.

68 Rim CH, Kim CY, Yang DS, Yoon WS. Comparison of radiation therapy modalities for hepatocellular carcinoma with portal vein thrombosis: a meta-analysis and systematic review. Radiother Oncol. 2018;129(1):11222.

69 Network NCC. NCCN Clinical Practice Guidelines in Oncology: Hepatobiliary cancers. Version 2.2015; 2015

70 Ben-Josef E, Normolle D, Ensminger WD, Walker S, Tatro D, Ten Haken RK, et al. Phase II trial of high-dose conformal radiation therapy with concurrent hepatic artery floxuridine for unresectable intrahepatic malignancies. J Clin Oncol. 2005;23(34):8739-47.

71 Sato K, Lewandowski RJ, Bui JT, Omary R, Hunter RD, Kulik L, et al. Treatment of unresectable primary and metastatic liver cancer with yttrium-90 microspheres (TheraSphere ${ }^{\oplus}$ ): assessment of hepatic arterial embolization. Cardiovasc Intervent Radiol. 2006;29(4):522-9.

72 Wang TH, Huang PI, Hu YW, Lin KH, Liu CS, Lin YY, et al. Combined Yttrium-90 microsphere selective internal radiation therapy and external beam radiotherapy in patients with hepatocellular carcinoma: from clinical aspects to dosimetry. PLoS One. 2018;13(1): e0190098.

73 Elschot M, Vermolen BJ, Lam MG, de Keizer B, van den Bosch MA, de Jong HW. Quantitative comparison of PET and Bremsstrahlung SPECT for imaging the in vivo yttrium-90 microsphere distribution after liver radioembolization. PLoS One. 2013;8(2):e55742.

74 Weng Z, Ertle J, Zheng S, Lauenstein T, Mueller S, Bockisch A, et al. Choi criteria are superior in evaluating tumor response in patients treated with transarterial radioembolization for hepatocellular carcinoma. Oncol Lett. 2013;6(6):1707-12.

75 Jongen JMJ, Rosenbaum CENM, Braat MNGJA, van den Bosch MAAJ, Sze DY, Kranenburg $\mathrm{O}$, et al. Anatomic versus metabolic tumor response assessment after radioembolization treatment. J Vasc Interv Radiol. 2018; 29(2):244-e2

76 Shim JH, Lee HC, Kim SO, Shin YM, Kim KM, Lim YS, et al. Which response criteria best help predict survival of patients with hepatocellular carcinoma following chemoembolization? A validation study of old and new models. Radiology. 2012;262(2):70818.

77 Jung ES, Kim JH, Yoon EL, Lee HJ, Lee SJ, Suh SJ, et al. Comparison of the methods for tumor response assessment in patients with hepatocellular carcinoma undergoing transarterial chemoembolization. J Hepatol. 2013; 58(6):1181-7.

78 Kennedy AS, Ball DS, Cohen SJ, Cohn M, Coldwell DM, Drooz A, et al. Hepatic imaging response to radioembolization with yttrium90-labeled resin microspheres for tumor progression during systemic chemotherapy in patients with colorectal liver metastases. J Gastrointest Oncol. 2015;6(6):594.

79 Guglielmi A, Ruzzenente A, Campagnaro T, Pachera S, Valdegamberi A, Nicoli P, et al. Intrahepatic cholangiocarcinoma: prognostic factors after surgical resection. World J Surg. 2009;33(6):1247-54.

80 Endo I, Gonen M, Yopp AC, Dalal KM, Zhou $\mathrm{Q}$, Klimstra D, et al. Intrahepatic cholangiocarcinoma: rising frequency, improved survival, and determinants of outcome after resection. Ann Surg. 2008;248(1):84-96.

81 Mavros MN, Economopoulos KP, Alexiou VG, Pawlik TM. Treatment and prognosis for patients with intrahepatic cholangiocarcinoma: systematic review and meta-analysis. JAMA Surg. 2014;149(6):565-74.

82 Lieser MJ, Barry MK, Rowland C, Ilstrup DM, Nagorney DM. Surgical management of intrahepatic cholangiocarcinoma: a 31-year experience. J Hepatobiliary Pancreat Surg. 1998; 5(1):41-7.

83 Edeline J, Touchefeu Y, Guiu B, Farge O, Tougeron D, Baumgaertner I, et al. Radioembolization plus chemotherapy for first-line treatment of locally advanced intrahepatic cholangiocarcinoma: a phase 2 clinical trial. JAMA Oncol. 2020;6(1):51-9.

84 Schneider U, Pedroni E. Proton radiography as a tool for quality control in proton therapy. Med Phys. 1995;22(4):353-63.

85 Lamarca A, Ross P, Wasan HS, Hubner RA, McNamara MG, Lopes A, et al. Advanced intrahepatic cholangiocarcinoma: post hoc analysis of the ABC-01,-02, and- 03 clinical trials. JNCI. 2020;112(2):200-10. 